\title{
Structural Investigation on Thermoresponsive PVA/ Poly(methacrylate-co- $N$-isopropylacrylamide) Microgels across the Volume Phase Transition
}

\author{
Shivkumar V. Ghugare, ${ }^{\dagger}$ Ester Chiessi, ${ }^{\dagger}$ Rainer Fink, ${ }^{\dagger}$ Yuri Gerelli, ${ }^{\perp}$ Andrea Scotti, ${ }^{\S}$ Antonio Deriu, ${ }^{\S}$ \\ Geraldine Carrot," and Gaio Paradossi ${ }^{\dagger, *}$ \\ ${ }^{\dagger}$ Dipartimento di Scienze e Tecnologie Chimiche, Università di Roma Tor Vergata, 000133 Roma, Italy \\ ${ }^{\ddagger}$ Physikalische Chemie II and ICMM, Friedrich-Alexander Universität Erlangen-Nürnberg, Egerlandstrasse 3, \\ D-91058 Erlangen, Germany \\ ${ }^{\S}$ Dipartimento di Fisica, Università di Parma, Parma, Italy \\ ${ }^{\perp}$ Institute Laue Langevin, 6 rue Jules Horowitz, 38000 Grenoble, France \\ "Laboratoire Léon Brillouin, CEA Saclay, 91191 Gif-sur-Yvette Cedex, France
}

Supporting Information

\begin{abstract}
Characterization of switchable microgels is a major task in drug delivery science. The study of soft polymeric devices requires a combined use of spectroscopy, microscopy, and scattering approaches enabling the characterization of nanostructured features across a volume phase transition. In this work the structural changes of poly(vinyl alcohol) based thermoreversible microgel particles which incorporate $\mathrm{p}(\mathrm{NiPAAm}$-co-methacrylate) chains across the transition temperature occurring at $33{ }^{\circ} \mathrm{C}$ have been addressed by utilizing reciprocal and direct space approaches such as small angle neutron scattering, SANS, dynamic light scattering, DLS, soft transmittance X-ray microscopy, STXM, and confocal laser scanning microscopy, CLSM, respectively. The

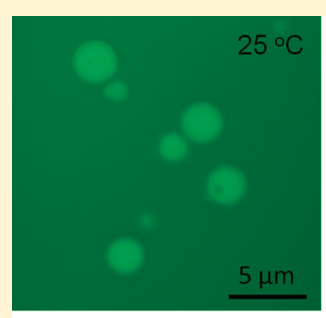

Permeable

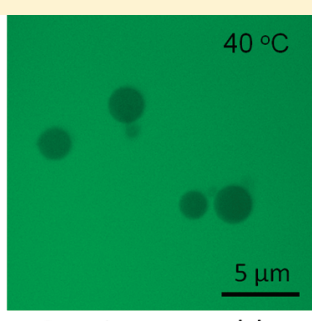

Semi-permeable comparison between the results obtained from those approaches allows an evaluation of the driving forces acting in the transition and reveals the changes in the microgel structure at nanoscale level. The structure of the poly(vinyl alcohol) based microgel particles, incorporating $\mathrm{p}(\mathrm{NiPAAm})$ sequences, consist of a hydrogel core and of a crown of polymer chains projected, at room temperature, in aqueous medium. An increase of the temperature above $33^{\circ} \mathrm{C}$ causes a volume phase transition of the system characterized by the collapse of the particle core and of the chains grafted at the particle surface. This transition is accompanied by a massive release of water and an increase of the interface with the dispersing aqueous medium, causing the passage from a permeable to a semipermeable structure.
\end{abstract}

\section{INTRODUCTION}

Switchable hydrogels were described first in the inspiring works of Tanaka in the eighties. ${ }^{1-8}$ It is now clear that "soft", polymer based, switchable systems can be a powerful answer to the several requirements raised in modern drug delivery, ${ }^{9}$ transducer technologies, ${ }^{10}$ and biotechnologies. ${ }^{11}$ Changes in the physical state of monolithic hydrogels were already studied and rationalized by Flory within the framework of the Flory-Rehner theory, ${ }^{12-14}$ an extension of the well-known theory on polymer mixing process. ${ }^{14}$ Further insight was provided by considering gels as fractal systems. ${ }^{15}$ Issues such as responsivity to physiological changes of $\mathrm{pH}$ and temperature, localized drug delivery, biodegradability, bioadhesion, hydration can be matched, in principle, with biocompatible colloidal dispersions of polymeric micro- or nanoparticles. In the past decade attention of the researchers has moved to microgels, i.e., systems able to combine the features of macroscopic hydrogels such as connectivity and response to external stimuli with the colloidal properties of nano/micro sized systems. Because of their large specific surface, these systems enable a variety of surface modifications. Moreover, moving from macro- to microscopic gels allows a systemic administration of the drug loaded microgel, a decrease in time response to an external trigger as $\mathrm{pH}$ or temperature changes and the exploitation of this feature for a fast and targeted delivery of the drug. In switchable microgels designed for the controlled drug release, the combination of the colloidal properties with injectability for a systemic administration of a large drug loading capacity and biocompatibility is a requirement for potential application.

Thermoresponsivity is one of the most studied features for a shrinking-assisted drug delivery. The great majority of the studies addressing the design of thermoresponsive systems finalized to the release of a drug were dealing with $N$-isopropylacrylamide, NiPAAm, based polymers, ${ }^{16}$ copolymers, ${ }^{17}$ hydrogels, ${ }^{18}$ and nanogels/microgels. ${ }^{19-22}$ Hydrophilic polymer systems incorporating

Received: March 22, 2011

Published: May 11, 2011 
Scheme 1. Structure of the PVA/Poly(methacrylate-coNiPAAm), PMN-II, Network $(m=0.05 ; n=0.12)$

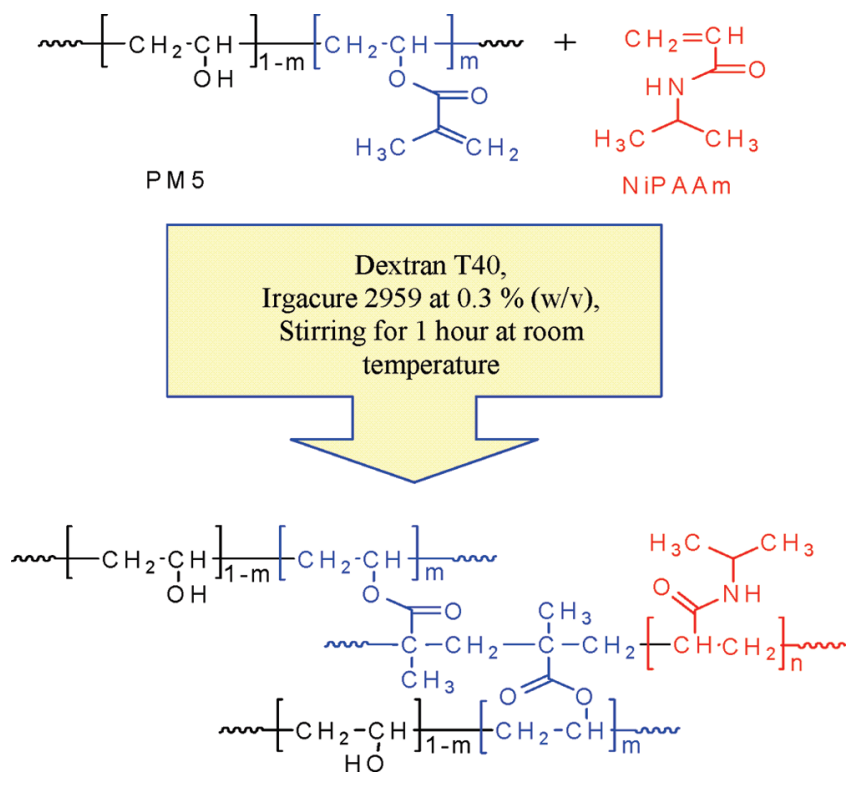

residues with lower critical solution temperature (LCST) close to physiological temperature can be considered as thermoresponsive systems. Recently, we reported on stable thermoresponsive microgels based on soluble poly(vinyl alcohol) grafted with methacryloyl side chains with a degree of substitution (DS) of $5 \%$, PM5. ${ }^{23,24}$ Water-in-water microemulsion technique ${ }^{25}$ can be applied to PM5 aqueous solutions using aqueous dextran T40 as dispersion phase. The immiscibility of the two aqueous phases offers the possibility to photopolymerize the emulsion with a separation of microgel particles. ${ }^{25,26}$ Addition of NiPAAm and photopolymerization of the emulsion allows the incorporation of this residue in a PVA/poly(methacrylate-co-NiPAAm), PMN-II, network (see Scheme 1). ${ }^{27}$

Furthermore, we addressed the dynamic behavior of water associated with the network probing the relaxation processes occurring in the microgels by QENS. ${ }^{29}$ Here we focus on how the microgel-water interface changes on the micro/nanoscopic level upon a temperature-driven volume phase transition, using direct and reciprocal space investigation methods having negligible impact on the polymer soft matter devices. Confocal laser scanning microscopy (CLSM) coupled with a permeation study provided a pore size evaluation across the transition temperature. Scanning transmission X-ray microscopy (STXM) was used for the evaluation of the overall size of individual particles in wet condition at high spatial resolution. The momentum transfer values probed by small angle neutron scattering (SANS) allowed to exploring the surface behavior and the pore size of the microgels across the transition. The picture emerging from these approaches is compared with the dynamic light scattering results disclosing the role of interfacial water across the VPTT.

\section{EXPERIMENTAL SECTION}

Materials. N-Isopropylacrylamide (NiPAAm) purchased from Aldrich, was recrystallized in $n$-hexane prior to use. All other chemicals were used as received. Poly(vinyl alcohol), PVA, with number and weight-average molecular weights of $30000 \pm 5000$ and $70000 \pm$ $10000 \mathrm{~g} / \mathrm{mol}$, respectively, dextranT40 with average molecular weight of $35000-40000 \mathrm{~g} / \mathrm{mol}$, and $\mathrm{D}_{2} \mathrm{O}$ were purchased from Sigma-Aldrich. 4-(N,N-Dimethylamino)pyridine (DMAP), glycidyl methacrylate (GMA) and fluorescein isothiocynate isomer 1 (FITC) were Fluka products. Photoinitiator 2-hydroxy-1-[4-(hydroxyethoxy)phenol]-2methyl 1-propanone (Irgacure 2959) was purchased from Ciba. Dimethyl sulfoxide (DMSO), inorganic acids, and bases were RPE grade products supplied by Carlo Erba (Italy).

Water was Milli-Q purity grade $(18.2 \mathrm{M} \Omega \cdot \mathrm{cm})$ produced with a deionization apparatus (PureLab) from USF, Elga. Dialysis membranes (cutoff $12000 \mathrm{~g} / \mathrm{mol}$ ) were purchased from Medicell International Ltd. and prepared according to standard procedure.

Methods. Preparation of PVA-Based Thermoresponsive Microgels. According to the procedure described elsewhere. ${ }^{25,28-30}$ PVA backbone was grafted with glycidyl methacrylate with a degree of substitution of $5 \%$. Microgels based on 5\% methacrylated PVA (PM5) were prepared, adapting a procedure originally introduced by Franssen and Hennink, ${ }^{25}$ which employs a water-in-water emulsion technique based on polymer-polymer immiscibility in aqueous solution. In a typical experiment, an aqueous dispersion containing dextran T40 at a concentration of $16 \%$ $(\mathrm{w} / \mathrm{v})$, PM5 at $2 \%(\mathrm{w} / \mathrm{v}), 1.3 \%$ NiPAAm $(\mathrm{w} / \mathrm{v})$, and the UV photoinitiator Irgacure 2959 at $0.3 \%(\mathrm{w} / \mathrm{v})$ was vigorously stirred by an UltraTurrax emulsifier at $16000 \mathrm{rpm}$. After emulsification, PM5 in the dispersed aqueous phase was cross-linked by photopolymerization using a $365 \mathrm{~nm}$ light source at an intensity of $7 \mathrm{~mW} / \mathrm{cm}^{2}$ for $5 \mathrm{~min}$. The crosslinked PVA/poly(methacrylate-co- $N$-isopropylacrylamide) microgels were purified by repeated steps of centrifugation and resuspended in Milli-Q water. The effective amount of NiPPAm incorporated in the network (NiPPAm/methacrylate molar ration: 2.4 and NiPPAm weight fraction: $22 \%(\mathrm{w} / \mathrm{w}))$ were determined by elemental analysis. ${ }^{24}$ Here after the obtained microgels were labeled as PMN-II.

Scanning Electron Microscopy (SEM). The morphology of the microgel particles was probed by scanning electron microscopy (Zeiss Supra VP55) high-resolution field emission scanning electron microscope at the Paul Scherrer Institute (PSI, Villigen, Switzerland). Diluted dispersions of microgel particles were dropped onto mica substrates and dried at room temperature in air prior to observation.

Dynamic Light Scattering (DLS). Evaluation of the average hydrodynamic diameter and size distribution of the microgels was carried out by dynamic light scattering (DLS) experiments using a BI-200SM goniometer (Brookhaven Instruments Co.) equipped with a solid state laser source emitting at $532 \mathrm{~nm}$ with an external water bath thermostat. The temperature was controlled from 20 to $43{ }^{\circ} \mathrm{C}$ with an accuracy of $\pm 0.2 \mathrm{deg}$. The autocorrelation functions were analyzed with a standard software package using cumulant analysis to obtain the hydrodynamic diameter of the microgels and the size distribution from the linear, $\Gamma$, and quadratic, $\mu_{2}$, terms of the power series expansion, respectively:

$$
\ln \left[g^{(1)}(\tau)\right]=-\bar{\Gamma} \tau+\frac{\mu_{2}}{2 !} \tau^{2}-\frac{\mu_{3}}{3 !} \tau^{3}+\frac{\mu_{4}}{4 !} \tau^{4}+\ldots
$$

Differential Scanning Calorimetry (DSC). Volume phase transition temperature of the PMN-II microgels was examined using a TA Q2000 differential scanning calorimeter. Known amounts of dry microgels were equilibrated in Milli-Qwater at room temperature to reach the maximum swelling and centrifuged to eliminate the excess of water. An exactly weighted amount of slurry, about $20-30 \mathrm{mg}$, was placed inside an aluminum pan and then hermetically sealed with an aluminum lid. The scans were performed from 25 to $55{ }^{\circ} \mathrm{C}$ on the swollen microgels at a heating rate of $3{ }^{\circ} \mathrm{C} / \mathrm{min}$ under a flux of $50 \mathrm{~mL} / \mathrm{min}$ of dry nitrogen.

Confocal Laser Scanning Microscopy (CLSM). Freeze-dried microgels were first immersed in Milli-Q water at room temperature to reach their swollen state. The swollen microgels were labeled with FITC by coupling the dye to the hydroxyl group of microgels. ${ }^{31}$ Fluorescent dye at a concentration of $10 \mu \mathrm{M}$ was added in the dispersion. The unreacted 


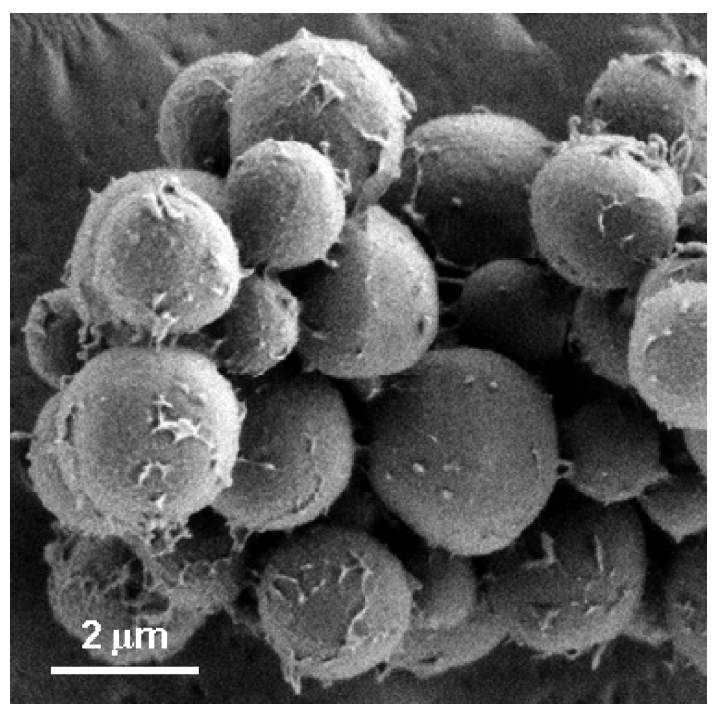

Figure 1. SEM micrograph of PMN-II microgels in the dry state.

dye was removed by repeated washings with Milli-Q water, and CLSM observations were performed using a confocal laser scanning microscope (Nikon PCM 2000, Nikon Instruments, Japan) with a Plan Apo 60XA/ 1.4 oil immersion objective. Images were collected with a pixel size of $0.414 \mu \mathrm{m}$ using the entire field of $512 \times 512$ pixels.

For temperature controlled measurements a Bioptechs FCS2 and FCS 3 closed chamber stage with a built-in temperature control unit was used at room temperature and at $40{ }^{\circ} \mathrm{C}$, following the same particles below and above the VPTT. The evaluation of average microgel diameter was carried out considering only the particles with the equatorial plane on focus.

Scanning Transmission X-ray Microscopy (STXM). For STXM measurements we used the so-called "wet cells" where approximately $1 \mu \mathrm{L}$ of well homogenized microgel water suspension was sandwiched between two $100 \mathrm{~nm}$ thick $\mathrm{Si}_{3} \mathrm{~N}_{4}$ membranes (Silson Ltd., U.K.), which were then sealed with silicone high-vacuum grease to maintain the water environment during the experiment. The microgels were imaged in transmission mode in helium atmosphere at the PolLux-STXM beamline at the Swiss Light Source (SLS), using the synchrotron radiation from the electron storage ring at the Paul Scherrer Institute (Villigen, Switzerland). ${ }^{32}$ The transmitted photon flux was measured using a photomultiplier tube (Hamamatsu 647P). The PolLux-STXM uses linearly polarized $\mathrm{X}$-rays from a bending magnet in the photon energy range between 260 and $1100 \mathrm{eV}$ and it routinely provides a spatial resolution better than $40 \mathrm{~nm}^{33}$

Images were recorded at selected energies through the $\mathrm{O} 1 \mathrm{~s}$ region $(510-560 \mathrm{eV})$. The data for the beam-induced changes of the microgels were obtained during a normal "line-by-line" imaging experiment with a dwell time of $1 \mathrm{~ms}$ (data integration time per one image pixel). Carbon K-edge NEXAFS spectra were collected in line mode, i.e., the transmitted signal was recorded while a line trajectory was scanned across the center of microgel particle at each value of the photon energy through the spectrum. Data processing was carried out using the aXis2000 software. ${ }^{34}$ Radial transmittance profile processing was performed with homemade software.

Small Angle Neutron Scattering (SANS). SANS experiments of thermoresponsive microgels were performed using the PAXE diffractometer at Laboratoire Leon Brillouin facility, LLB, ${ }^{35}$ as a function of temperature $\left(29,32,35,37\right.$, and $\left.42{ }^{\circ} \mathrm{C}\right)$. The temperature of the chamber was controlled by circulating water with the precision of $\pm 0.5^{\circ} \mathrm{C}$. In order to cover a large $Q$-range, $6 \AA$ wavelength neutrons were used with two instrumental configurations: the $64 \times 64 \mathrm{~cm}^{2}$ gas filled detector, having a resolution of $1 \mathrm{~cm}^{2}$, was placed $1.3 \mathrm{~m}$ apart from

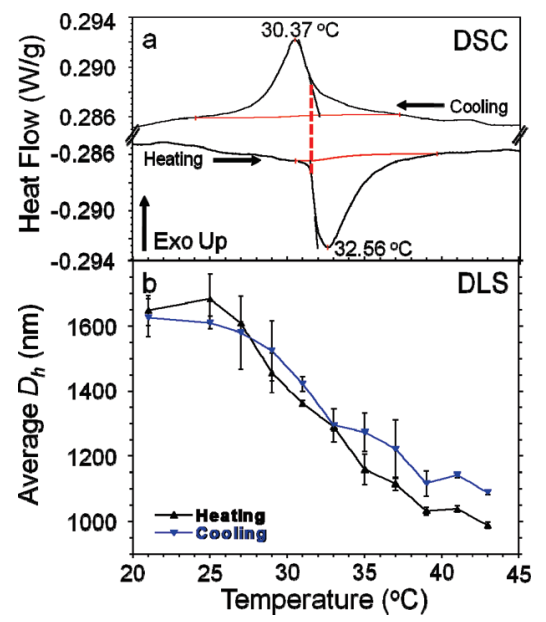

Figure 2. DSC thermogram (a) and average hydrodynamic diameter derived from DLS (b) of PMN-II microgels revealing the temperature behavior upon cooling and heating. Dashed line evidence the onset of the transition upon heating and cooling. Microgel dispersed in $\mathrm{H}_{2} \mathrm{O}$.

the sample for large angles and $5 \mathrm{~m}$ apart for small angles. The resultant Q-range was $8.5 \times 10^{-3}$ to $3.0 \times 10^{-1} \AA^{-1}$. Standard corrections, cell subtraction and normalization to absolute scattering units have been performed using routines provided by the LLB facility. All samples were dispersed in $\mathrm{D}_{2} \mathrm{O}$ at a polymer fraction of $10 \%(\mathrm{w} / \mathrm{v})$ and placed in $1 \mathrm{~mm}$ path Hellma quartz cells, prior to undertake the measurements.

\section{RESULTS AND DISCUSSION}

Figure 1 shows a scanning electron microscopy (SEM) micrograph of PMN-II microgels in the dry state.

In this agglomerate the microgel particles display an almost spherical morphology with quite homogeneous particle sizes around an average diameter of $2 \mu \mathrm{m}$. These findings, collected on a dry microgel sample, are in good agreement with average particles dimension and size distribution obtained at room temperature in solution by dynamic light scattering. The SEM image reveals some protrusions over a generally smooth surface. Recently we have investigated on the chemical structure and on the thermal responsivity of these microgel particles consisting in a volume decrease around a critical temperature. The "water-inwater" emulsion method, used for the fabrication of PMN-II microgel particles, implies the presence of NiPAAm monomers in both aqueous phases. This leads to the obtainment of microgel particles with an inner, more dense and cross-linked, part incorporating NiPAAm residues and an outer part, with linearly polymerized NiPAAm sequences. Therefore, it can be expected that both parts will contribute to the thermoresponsivity of the microgel particle. The thermal behavior of these microgel particles is expected to be different from the "core-shell" particles described in the literature, having the temperature responsive part confined in the shell, with a core inert to temperature. ${ }^{36}$

A lower critical solution temperature, LCST, is common to all polymers and oligomers containing sequence of more than 3-4 NiPAAm residues. ${ }^{37-39}$ Differential scanning calorimetry (Figure $2 \mathrm{a}$ ) of PMN-II shows the occurrence of an endothermic, entropy driven transition at $32.6{ }^{\circ} \mathrm{C}$ accompanied by a hydrodynamic diameter reduction by about $30 \%$ as determined from dynamic light scattering (see Figure $2 \mathrm{~b}$ ). This volume phase transition can be rather sharp depending on the degree of connectivity of the network and it is reminiscent of the LCST 

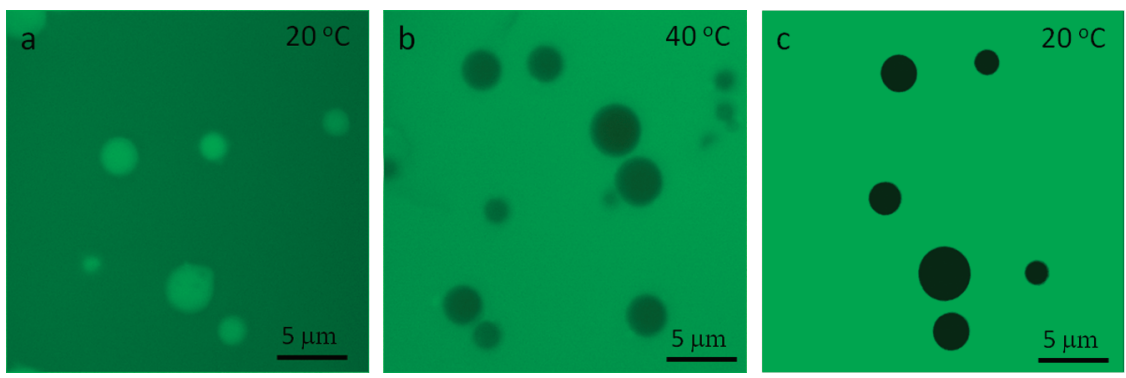

Figure 3. Confocal laser scanning microscopy images of PVA based thermoresponsive (PMN-II) microgels a) at room temperature $\left.\left(20^{\circ} \mathrm{C}\right), \mathrm{b}\right)$ at $40{ }^{\circ} \mathrm{C}$ with FD 4, FITC-Dextran (average MW $4 \mathrm{~kg} / \mathrm{mol}$ ) respectively and c) at room temperature $\left(20^{\circ} \mathrm{C}\right)$ with FD 10, FITC-Dextran (average MW $\left.10 \mathrm{~kg} / \mathrm{mol}\right)$.

behavior of poly(NiPAAm) chains in aqueous solution with a critical temperature of about $32{ }^{\circ} \mathrm{C} .^{38}$

The detailed architecture of the microgel particles, as the pore size of the network, was subjected to a remarkable structural decrease across the volume phase transition temperature. This process was evidenced by size exclusion/confocal laser scanning microscopy (SE/CLSM) and semiquantitatively assessed. In Figure 3 a summary of the main structural changes is reported. ${ }^{27}$

In the following sections we discuss the results provided by different approaches, namely small angle neutron scattering and $\mathrm{X}$-ray microscopy, in an effort to have a deeper insight of the processes occurring in the microgels across the VPTT. The use of SANS addresses the structural features of the internal architecture of the microgel particles, as the average pore size, whereas a real space method such as X-ray microscopy is used for the study of the changes in the overall dimensions of the particles at higher resolution than that one provided by confocal microscopy, but preserving the wet conditions necessary for the volume phase transition of the microgel.

SANS is a widely used technique for investigating the detailed structures of polymer hydrogels and microgels. ${ }^{40-44}$ SANS experiments consist of the measurement of the scattered intensity $I(Q)$ as a function of the scattering vector $Q(Q=(4 \pi / \lambda) \sin$ $(\theta / 2)$, where $\lambda$ is the wavelength and $\theta$ the scattering angle). The scattering intensity $I(Q)$ is obtained by subtracting a flat incoherent background $I(Q)_{i n c}$ from the observed intensity $I(Q)_{\text {obs }}$.

The scattering intensity for gel systems is often expressed by one or more Ornstein-Zernike-like functions. ${ }^{45} I_{\text {fluct }}(Q)$ accounting for the scattering from the fluctuations of the network and $I_{\text {stat }}(Q)$ describing the static heterogeneities of the network.

$$
\begin{gathered}
I_{\text {fluct }}(Q) \propto \Delta \rho^{2} \frac{k_{\mathrm{B}} T}{1+Q^{2} \xi^{2}} \\
I_{\text {stat }}(Q) \propto \Delta \rho^{2} \frac{\Xi^{3}}{\left[1+Q^{2} \Xi^{2}\right]}
\end{gathered}
$$

where $\Delta \rho$ is the difference between the scattering length density of the polymer and of the solvent, $\mathrm{D}_{2} \mathrm{O}$ in the present experiment. $\xi$ and $\Xi$ are respectively the correlation lengths describing thermal fluctuations and heterogeneities of the polymer network. The final expression for the scattering intensity is obtained convoluting the sum of terms 2 and 3 with the instrumental resolution $R(Q)$ :

$$
I(Q)=\left[I_{\text {fluct }}(Q)+I_{\text {stat }}(Q)\right] \otimes R(Q)
$$

Since our particles are micrometer-sized and considering the investigated $Q$-range, we can neglect the contribution to the scattering intensity due to the whole particle (arising from the so-called form factor). Nevertheless using the Porod approximation ${ }^{46,47}$ in the low- $Q$ region we can extract important information on the surface features of the scatterers, in analogy with the work of Fernàndez-Barbero et al. on pNiPAAm microgels. ${ }^{48}$

In this frame the $I_{\text {stat }}(Q)$ term can be approximated by:

$$
I_{\text {stat }}(Q) \propto Q^{-\alpha}
$$

Three-dimensional objects, $d=3$, with a fractal surface are described by the characteristic dimension $D_{\mathrm{S}}$, whose value is bound between 2 and 3, i.e. $(d-1)<D_{S}<d$. In this case, the scattering law will scale with $D_{\mathrm{S}}$ according to

$$
I_{\text {stat }}(Q) \propto Q^{-\left(2 d-D_{s}\right)}
$$

Equation 6 converges to the Porod's law when $D_{S} \rightarrow 2$, i.e. when $\alpha$ of eq 5 is equal to $4,{ }^{46,47}$ indicating a sharp interface between the three-dimensional object and the dispersion medium. In this treatment, the Porod's law is the limit case of the Q-dependence of the scattering intensity represented by a threedimensional isotropic object (dimensionality $d=3$ ), with a surface fractal dimension, $D_{S}=2$, corresponding to a smooth surface. Usually the surface of the microgel particle has a higher fractality described by a value of $D_{S}$ higher than 2 . In this case the scattering law is described by the decreasing power law, eq 4, with $\alpha<4$.

At higher $Q$ values the term $I_{\text {fluct }}(Q)$ in eq 4 prevails on $I_{\text {stat }}(Q)$, as the former decays following a $Q^{-2}$ law. This opens a clue on the detailed structure of the microgel network. In the limit $Q \xi<1$, the scattering law is dominated by $I(Q)_{\text {stat }}$, characterized by the correlation length, $\xi$, of the concentration fluctuations occurring in an isotropic semidilute polymer solution or of the average mesh size of a polymer network. Depending on the $Q$ values of choice, distances smaller than $\xi$ contribute to the correlation function, $G(r)$, according to: ${ }^{49}$

$$
G(r)=\langle\varphi(0) \cdot \varphi(r)\rangle \approx r^{-1} \exp \left(-\frac{r}{\xi}\right)
$$

Equation 7 establishes that the polymer concentration fluctuations can be considered as essentially uncorrelated on length scales larger than $\xi$. For a polymer in a good solvent, this means that the correlation length is roughly equal to the average distance between interchain contact points. ${ }^{50}$

Fourier transform of eq 7, in the limit $Q \xi<1$, yields the term $I(Q)_{\text {fluct }}$ of eq 2, in the form of a Ornstein-Zernike type function. ${ }^{45}$ When cross-links are introduced to a polymer solution, the concentration fluctuations are perturbed and the exact solution for the scattering function from gels has not been developed yet because of the complexity and heterogeneity of cross-linked polymer domains. SANS studies on gels ${ }^{51}$ and on 


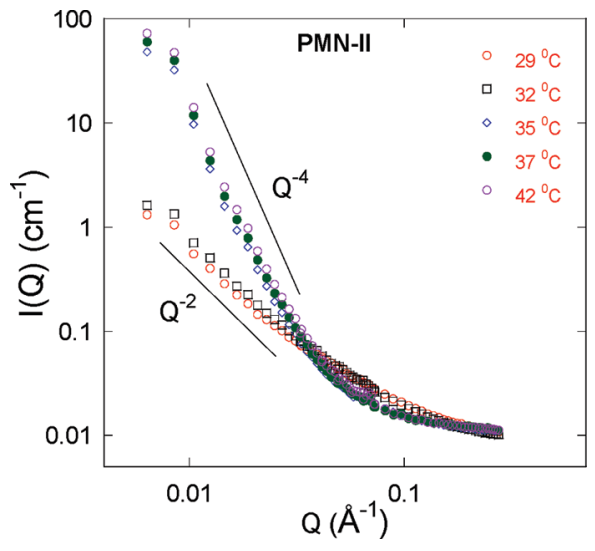

Figure 4. Double logarithmic plot of SANS intensity functions of PMN-II microgel at different temperatures.

semidilute polymer solutions ${ }^{52}$ show that the structural inhomogeneites of a network and the concentration fluctuation of a semidilute polymer solution can be described formally in terms of the Lorentzian function, eq $8 .{ }^{53}$ This approach leads to reformulate eq 4 as

$$
I(Q)=A Q^{-\alpha}+B \frac{I(0)}{\left[1+(\xi Q)^{2}\right]}
$$

Small-angle neutron scattering of PMN-II microgels was recorded at 29, 32, 35, 37, and $42{ }^{\circ} \mathrm{C}$. Figure S1 in Supporting Information shows the intensity profiles of the microgels from 29 to $42{ }^{\circ} \mathrm{C}$ on $x-y$ plane as color images (brighter color corresponding to higher scattering intensities) and indicating a totally disordered colloidal system.

The analysis of the scattered intensity profile shows (see Figure 4) a monotonically decreasing trend with increasing value of the scattering vector, $Q$ as we are exploring distances much lower than the overall microgel size. The SANS spectra are invariably composed by two regions for all the examined temperatures. At low $Q$ values, the scattered intensity increases with increasing temperature, approaching, for temperatures above the VPTT, the Porod scattering law, $\propto Q^{-4}$. At larger $Q$ values, where smaller distances are probed, differences between low and high temperature can be interpreted as a change in the correlation length $\xi$.

The data analysis of the low $Q$ regime was performed according to eq 5 , obtaining information about the solvent microgel particle interface. The trend of the parameter $\alpha$ as a function of the temperature is reported in Figure 5.

The exponent $\alpha$, i.e. the slope of the $\log -\log$ plot in Figure 5, depends strongly from the temperature, showing an abrupt increase around the VPTT. This clearly indicates that the surface features of the microgels changes above and below VPTT. The power law of the $I(Q)$ vs $Q$ plot for the swollen particles below the VPTT, has a value around $1.5-2$, indicating the presence of a microgel surface characterized by extended polymer branches. ${ }^{45}$ As the particles shrinks above the VPTT, $\alpha$ increases reaching values around 4 (Figure 5). Similar results on pNiPAAm microparticles were also found by Fernández-Barbero et al. ${ }^{48}$ and Kratz et al. ${ }^{54}$ who interpreted their data as a transition from a swollen surface with extended polymer chains in the solvent to a smooth surface of the microgel particles. This situation is representative also of the behavior of our microgels around the VPTT.

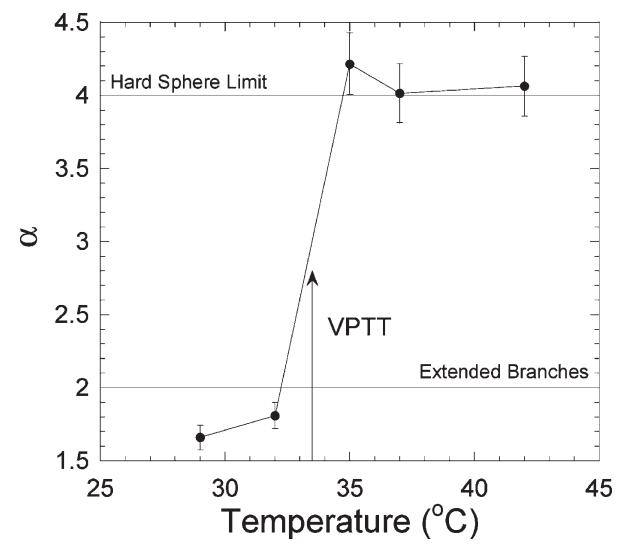

Figure 5. Exponent $\alpha$ as a function of temperature.

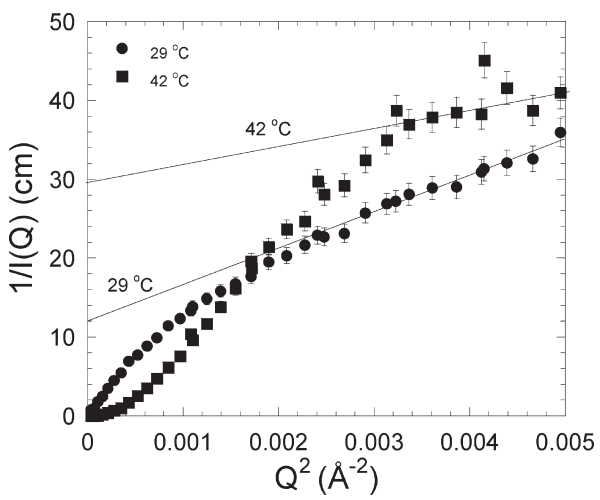

Figure 6. Ornstein-Zernike representation of the SANS spectra at 29 and $42{ }^{\circ} \mathrm{C}$. From the slope and intercept, the characteristic correlation length is determined for the PMN-II microgel.

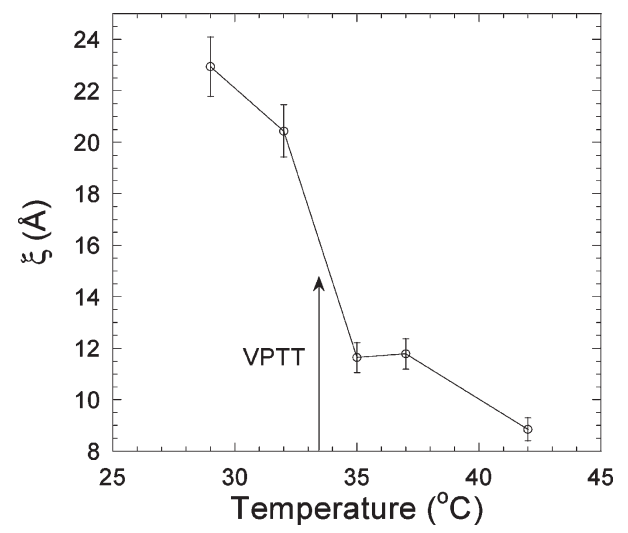

Figure 7. Temperature dependence of the characteristic correlation length for the PMN-II microgel.

The high Q region of SANS profiles provides further information on the network pore size across the volume phase transition and could be paired to the confocal microscopy/permeation studies already carried out on this system. ${ }^{27}$ In this regime the Lorentzian term of eq 9 prevails. The correlation length $\xi$ worked out for semidilute polymer solutions, was reinterpreted for gels as an average pore size dimension. An inverse plot of the $I(Q)$ vs $Q^{2}$ can be analyzed in terms of the linearized form of the 
Lorentzian function:

$$
\frac{1}{I(Q)}=\frac{1}{I(0)}+\frac{\xi^{2} Q^{2}}{I(0)}
$$

where the characteristic length $\xi$ is evaluated as the ratio of the square root of the slope over the intercept.

The analysis of this part of the $I(Q)$ according to eq 9 is shown in Figure 6 for room temperature and $42{ }^{\circ} \mathrm{C}$.

The correlation length $\xi$ across the VPTT varies from about 23 to $9 \AA$ with a sharp decrease at the transition temperature (see Figure 7).

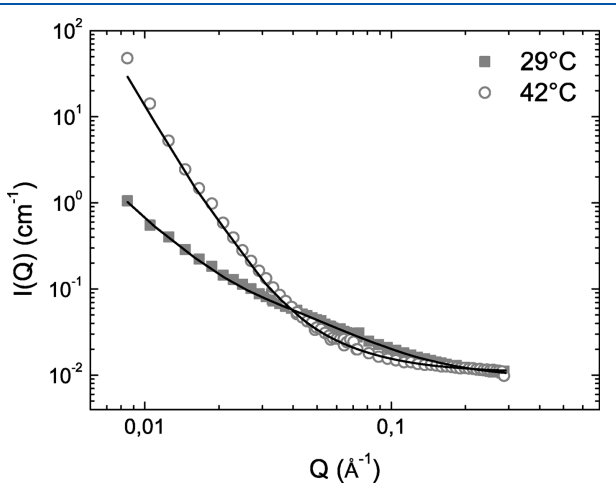

Figure 8. Scattering profiles for PMN-II samples below (solid squares) and above (open circles) the VPTT. Y-error bars are smaller than the size of the data points. Fits according to eq 4 are shown as solid lines.
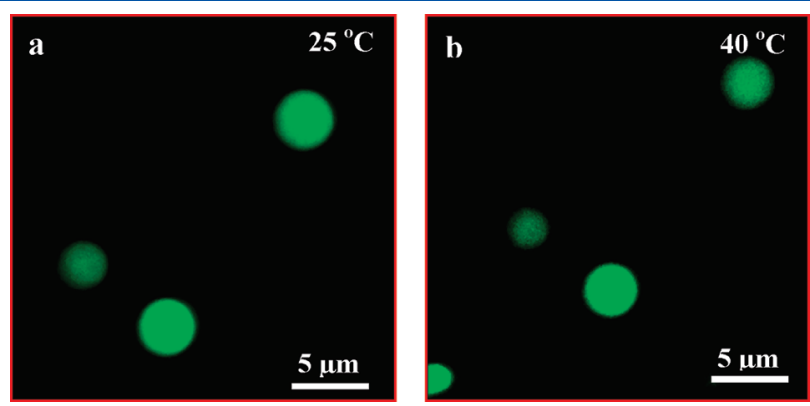

Figure 9. CLSM image of PMN-II microgels at 25 and $40{ }^{\circ} \mathrm{C}$.
This parameter reports on changes of the local domains in the microgel particles where $\mathrm{p}(\mathrm{NiPAAm})$ sequences included in the polymer network are in "extended" and in "collapsed" conformations below and above the volume phase transition temperature, respectively. This compares well with the evaluation of the microgel pore size carried out by size exclusion/CLSM experiments, ${ }^{27}$ where the pore size of the microgels was smaller than $5 \mathrm{~nm}$ at room temperature.

Alternatively, fit of the complete scattering profiles using eq 4 (see Figure 8) and adopting an instrumental resolution characterized by a $\Delta Q / Q=10 \%{ }^{55}$ yields values of the correlation length $\xi$ below and above VPTT in good agreement with the values reported in Figure 7. The second fitting parameter, $\Xi$, according to eq 3 , describes the structural heterogeneities within the gel. Its temperature dependence indicates an increase of the heterogeneities upon crossing the VPTT. This is directly related to the collapse of the microgel due to the establishment of hydrophobic domains in the microgel particles.

The volume phase transition of PMN-II was also investigated by means of scanning transmission X-ray microscopy (STXM). We have recently used this approach to assess the dimension, thickness and chemical composition of shell of PVA based microbubbles. ${ }^{56-59}$ Ade et al. ${ }^{60,61}$ have investigated the first direct imaging of electrolyte induced deswelling behavior of $\mathrm{pH}$-responsive microgels in aqueous media using STXM.

Here a similar approach has been employed for the thermoresponsive PMN-II microgels combining laser scanning confocal microscopy (CLSM) and scanning transmission X-ray microscopy (STXM).

In the Figure 9, CLSM image shows the direct shrinking behavior of single thermoresponsive PMN-II microgel and it reveals that the microgels particles shrink at $45^{\circ} \mathrm{C}$ by $16 \%$ of RT diameter. This study allows to assessing that above the volume phase transition temperature (VPTT) the surface area reduction ratio of PMN-II microgel is around $22 \%$.

The transition was monitored by STXM at $520 \mathrm{eV}$ and images of isolated microgel particles are exemplarily shown in Figure 10, parts $a$ and $b$, for two different temperatures, i.e., below and above the volume phase transition temperature, respectively.

The change of the radial transmittance profile as a function of temperature for the highlighted microgel particle is presented in Figure 10c, and it assesses shrinkage of the microgel particle at $45^{\circ} \mathrm{C}$ by $18 \%$ of its RT diameter. This behavior was common to all probed microgels particles. It is noteworthy that the change in
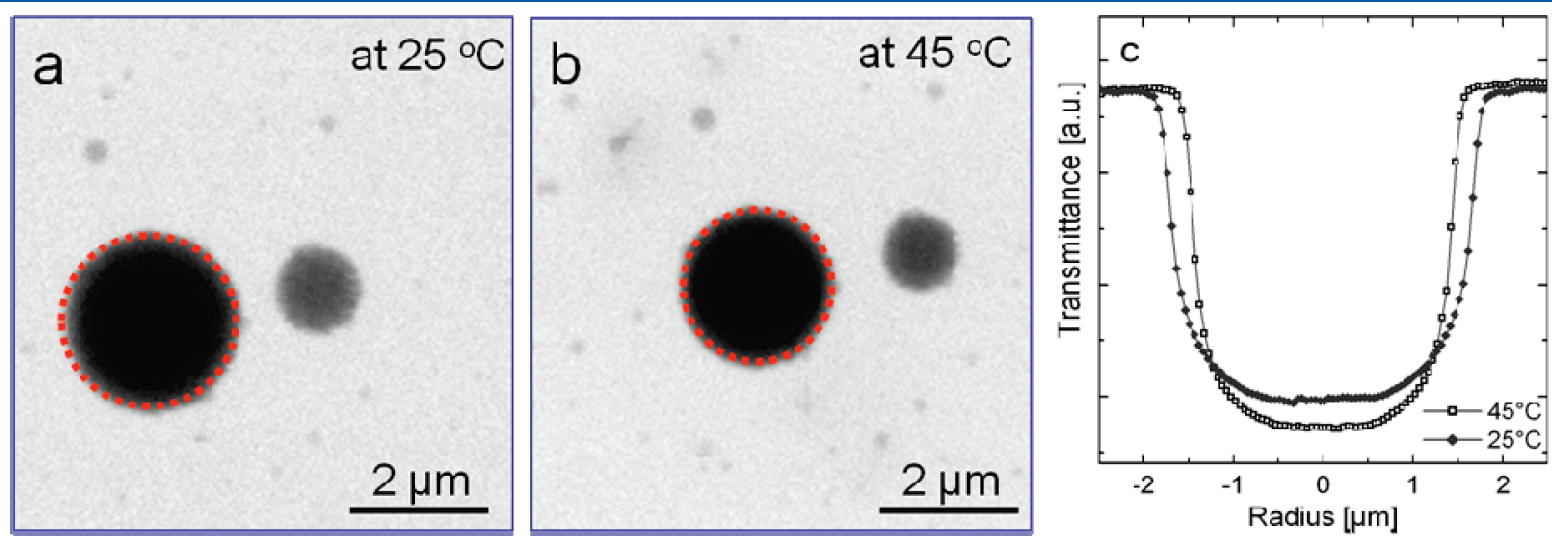

Figure 10. STXM transmission image at $25^{\circ} \mathrm{C}(\mathrm{a})$ and at $45^{\circ} \mathrm{C}(\mathrm{b})$. Radial transmittance profile of STXM images for the highlighted particle at 25 and $45^{\circ} \mathrm{C}(\mathrm{c})$. 
the slope of the radial profile upon temperature increase is an additional indication of a denser particle. We should note that the changes in the radial profiles and the overall reduction in transmittance upon heating lead to further conclusions. First, the decrease in transmittance for smaller particles clearly indicates much denser materials. Second, the changes in the slope in the outer region can only be explained by a densification of the material. Since STXM micrographs recorded at $520 \mathrm{eV}$ are basically sensitive to the optical thickness and density of the carbonaceous material, we may conclude on the reduction of water concentration in the respective area.

In order to rule out any chemical transformation of the microgel around the volume phase transition temperature (VPTT), the chemical composition of the microgels was probed

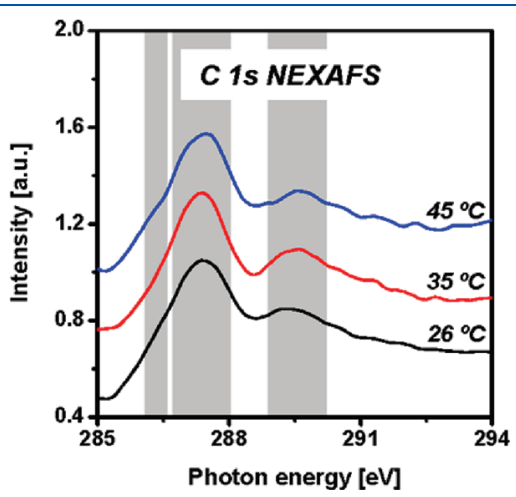

Figure 11. C 1s NEXAFS of PMN-II.

Table 1. Average Structural Parameters of PVA Based Thermoresponsive Microgels (PMN-II) by DLS, SANS, CLSM, and STXM below and above the Volume Phase Transition Temperature

$\begin{array}{cccccc} & \begin{array}{c}\text { pore size, } \\ \mathrm{nm}\end{array} & \begin{array}{c}\text { pore size, } \\ \mathrm{nm}\end{array} & \begin{array}{c}D_{h}, \mathrm{~nm} \\ (\mathrm{DLS})\end{array} & \begin{array}{c}\text { D, nm } \\ (\mathrm{CLSM})\end{array} & \begin{array}{c}\text { D, nm } \\ (\mathrm{STXM})\end{array} \\ \text { temperature } & (\mathrm{CLSM}) & (\mathrm{SANS}) & (\mathrm{C}) & & \\ & & 2.5 \pm 0.2 & 1650 \pm 200 & 1800 \pm 300 & 1600 \pm 100 \\ \text { below VPTT } & <5 & 1.0 \pm 0.1 & 900 \pm 100 & 1500 \pm 200 & 1300 \pm 200 \\ \text { above VPTT } & <3 & & \end{array}$

by carbon K-edge NEXAFS spectroscopy. The absorption spectrum of the microgel particles below and above VPTT is shown in Figure 11. Besides very minor intensity variations which are beyond the sensitivity of the technique, the T-dependent NEXAFS spectra confirm that the microgel chemical structure remains unchanged above the VPTT.

The structural changes in the microgel particle evaluated by four independent methods are summarized in Table 1.

In a perfect network, a reduction of a factor of 2 in the average pore size by crossing the VPTT, determined by SANS, would correspond to a 2 -fold decrease in the particle diameter. However, the microgel synthesis, based on emulsion polymerization, does not allow for a uniform cross-links density within the microgel particles ${ }^{62}$ and this aspect can justify the different variation occurring at nano- and micro- scales around the VPTT.

The change in hydrodynamic diameter of the microgel particles, evaluated by DLS, across the VPTT is $45 \%$ whereas the diameter difference measured directly by microscopy methods, i.e. on STXM and CLSM, is evaluated equal to $18 \%$. This apparent discrepancy between the diameter measured by DLS and the corresponding values obtained by CLSM and STXM above the VPTT can be explained considering the nature of the parameters measured with different approaches.

DLS reflects the shrinking of the particle core with the collapse of the extended chains crown on the particle surface, accompanied by a water depletion effect. On the contrary, this second contribution is scarcely detected by direct microscopies as STXM and CLSM, not sensible to the water loss of the particle crown. We have summarized in Chart 1 the differently weighting contributions to the diameter evaluation of the microgel particles carried out with DLS and direct methods as STXM and CLSM.

\section{CONCLUSIONS}

PVA is known for its hydrophilic character and is often used to maintain high hydration levels in devices when closely interacting with tissues. NiPAAm residues at room temperature are hydrophilic as well. The volume phase transition occurring at physiological temperature is due to the deswelling driven by the increase of hydrophobicity of NiPAAm in these conditions.

Chart 1. Schematic Representation of the Structural Changes Occurring in Thermoresponsive PMN-II Microgel Particles below and above the Volume Phase Transition Temperature (VPTT)
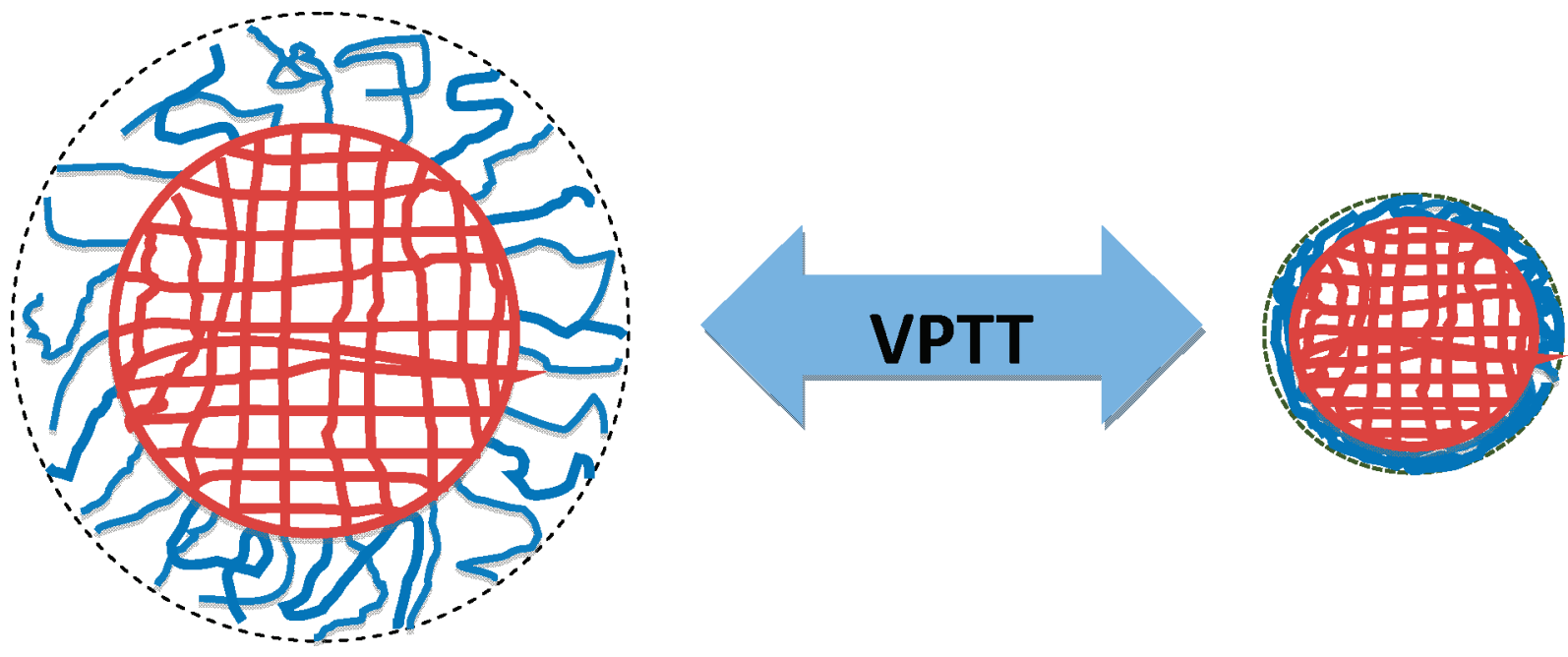
The incorporation of NiPAAm residues in the PVA based network induces a volume phase transition within a physiological temperature range. This was evidenced by confocal laser scanning microscopy (CLSM), since around the VPTT the pore size decreases from about 5 to $3 \mathrm{~nm} .{ }^{27}$ CLSM and STXM allows the direct observation of shrinking behavior of thermoresponsive microgels in aqueous solution. The shrinking behavior upon temperature increase is monitored in situ. Moreover, NEXAFS studies confirm that the microgel particles chemical structure remained unchanged above the volume phase transition temperature.

The fine structure of "smart" microgels has been studied by SANS. Increasing the scattered intensity of the SANS, above the volume phase transition temperature, the microgel surface smoothened significantly and reached to Porod profile. The change in pore size induced by temperature was revealed by the evaluation of the dependence of the correlation length. This result compares well with the findings obtained by previous size exclusion/CLSM experiments. A combined use of different scattering approaches probing several $Q$ decades, ranging from visible to $\mathrm{X}$-rays and thermal neutrons radiation, allowed a monitoring of the behavior of this thermoresponsive microgel across the VPTT. The cumulative amount of drug released from PMN-II particles above VPTT is enhanced with respect to the room temperature release. Considering that the loading of a positively charged drug, i.e., doxorubicin, was accomplished by introducing negative charges on the PVA moiety of the network, ${ }^{24}$ namely in the part of the microgel particles with higher chain density, we can conclude that the drug release is mainly due to this domain of the microgel. This background is necessary for the evaluation of the real potential of this delivery device for sustained drug release.

\section{ASSOCIATED CONTENT}

Supporting Information. Color images of SANS patterns from PVA/poly(methacrylate-co- $N$-isopropylacrylamide) microgel particles across the volume phase transition. This material is available free of charge via the Internet at http://pubs.acs.org.

\section{AUTHOR INFORMATION}

\section{Corresponding Author}

*Telephone: +3906 7259 4464. Fax: +3906 7259 4328. E-mail: paradossi@stc.uniroma2.it.

\section{ACKNOWLEDGMENT}

This work was partly funded by MIUR Project PRIN 20077LCNTW. We acknowledge Dr. George Tzvetkov for SEM images and assistance in part of the STXM experiments. S.V.G. was supported by the doctorate school of University of Rome Tor Vergata. The research leading to these results has received funding from the European Community's Seventh Framework Programme (FP7/2007-2013) under Grant Agreement No. 226716.

\section{REFERENCES}

(1) Tanaka, T.; Ishiwata, S.; Ishimoto, C. Phys. Rev. Lett. 1977, 38, 771.

(2) Tanaka, T. Phys. Rev. Lett. 1978, 40, 820.

(3) Tanaka, T.; Fillmare, D.; Sun, S.; Nishio, I.; Swislow, G.; Shah, A. Phys. Rev. Lett. 1980, 45, 163.
(4) Tanaka, T. Sci. Am. 1981, 244, 110.

(5) Tanaka, T.; Nishio, I.; Ueno-Nishio, S. Science 1981, 218, 467.

(6) Hirokawa, Y.; Tanaka, T. J. Chem. Phys. 1984, 81, 6379.

(7) Ricka, J.; Tanaka, T. Macromolecules 1984, 17, 2916.

(8) Suzuki, A.; Tanaka, T. Nature 1990, 346, 345.

(9) Langer, R; Peppas, N. AIChE 2003, 49, 2990.

(10) Jun, L.; Xia, H.; Yang, L. Y.; Di, L.; Yongwei, W.; Jinghong, L.; Ubai, B.; Tiejin, L. Adv. Mater. 2005, 17, 163.

(11) Pelton, R. Adv. Colloid Interf. Sci. 2000, 85, 1.

(12) Peppas, N.; Hilt, J.; Khademhosseine, A.; Langer, R. Adv. Mater. 2006, 18, 1345.

(13) Peppas, N.; Moynihan, H.; Lucht, L. J. Biomed. Mater. Res. 1985, $19,397$.

(14) Flory, P. J. Principles of Polymer Chemistry; Cornell University Press: Ithaca, NY, 1953.

(15) Courtens, E. J. Phys. IV 1992, 2, C2.

(16) Bawa, P.; Pillay, V.; Choonara, Y.; Toit, L. J. Biomed. Mater. 2009, 4, 022001.

(17) Kavanagh, C.; Rochev, Y.; Gallagher, W.; Dawson, K.; Keenan, A. Pharm. Therap. 2004, 102, 1.

(18) Qiu, Y.; Park, K. Adv. Drug Delivery Rev. 2001, 53, 321.

(19) Dai, X.; Chen, X.; Yang, L.; Foster, S.; Coury, A.; Jozefiak, T. Acta Biomater. 201110.1016/j.actbio.2011.01.005.

(20) Das, M.; Zhang, H.; Kumacheva, E. Annu. Rev. Mater. Res. 2006, $36,117$.

(21) Sanson, N.; Rieger, J. Polym. Chem. 2010, 1, 965.

(22) Lyon, L. A.; Meng, Z.; Singh, N.; Sorrell, C. D.; St John, A. Chem. Soc. Rev. 2009, 38, 865.

(23) Cavalieri, F.; Chiessi, E.; Villa, R.; Viganò, L.; Zaffaroni, N.; Telling, M. F.; Paradossi, G. Biomacromolecules 2008, 9, 1967.

(24) Ghugare, S. V.; Mozetic, P.; Paradossi, G. Biomacromolecules 2009, 10, 1589.

(25) Franssen, O.; Hennink, W. E. Int. J. Pharm. 1998, 168, 1.

(26) Stenekes, R.; Franssen, O.; van Bommel, E.; Crommelin, D.; Hennink, W. Pharm. Res. 1998, 15, 557.

(27) Ghugare, S.; Chiessi, E.; Telling, M.; Deriu, A.; Gerelli, Y.; Wüttke, J.; Paradossi, G. J. Phys. Chem. B 2010, 114, 10285.

(28) van Dijk-Wolthuis, W. N. E.; Franssen, O.; Talsma, H.; van Stenbergen, M. J.; Kettenes-van den Bosh, J. J.; Hennink, W. E. Macromolecules 1995, 28, 6317.

(29) van Dijk-Wolthuis, W. N. E.; Kettenes-van den Bosh, J. J.; van der Kerk-van Hoof, A.; Hennink, W. E. Macromolecules 1997, 30, 3411.

(30) Cavalieri, F.; Miano, F.; D’Antona, P.; Paradossi, G. Biomacromolecules 2004, 5, 2439.

(31) De Belder, A. N.; Granath, K. Carbohydr. Res. 1973, 30, 375.

(32) www.psi.ch/sls/pollux/pollux

(33) Raabe, J.; Tzvetkov, G.; Flechsig, U.; Böge, M.; Jaggi, A.; Sarafimov, B.; Vernooij, M. G. C.; Huthwelker, T.; Ade, H.; Kilcoyne, A. L. D.; Tyliszczak, T.; Fink, R. H.; Quitmann, C. Rev. Sci. Instrum. 2008, $79,113704$.

(34) Winn, B.; Ade, H.; Buckley, C.; Feser, M.; Howells, M.; Hulbert, S.; Jacobsen, C.; Kaznacheyev, K.; Kirz, J.; Osanna, A.; Maser, J.; McNulty, I.; Miao, J.; Oversluizen, T.; Spector, S.; Sullivan, B.; Wang, Y.; Wirick, S.; Zhang, H. J. Synchrotron Radiat 2000, 7, 395.

(35) www-llb.cea.fr/en/fr-en/pdf/paxe-llb.pdf

(36) Lu, Y.; Ballauf, M. Prog. Polym. Sci. 201110.1016/j.progpolymsci.2010.12.003.

(37) Schild, H. G. Prog. Polym. Sci. 1992, 17, 163.

(38) Graziano, G. Int. J. Biol. Macromol. 2000, 27, 89.

(39) Wang, X.; Qiu, X.; Wu, C. Macromolecules 1998, 31, 2972.

(40) Shibayama, M. Macromol. Chem. Phys. 1998, 199, 1.

(41) Shibayama, M.; Tanaka, T. J. Chem. Phys. 1992, 97, 6842.

(42) Shibayama, M.; Tanaka, T. J. Chem. Phys. 1992, 97, 6829.

(43) Stieger, M.; Richtering, W.; Pedersen, J.; Lindner, P. J. Chem. Phys. 2004, 120, 6197.

(44) Mason, T.; Lin, M. Phys. Rev. E 2005, 71, 040801.

(45) Higgins, J. S.; Benoit, H. C. Polymers and Neutron Scattering; Oxford University Press: New York, 1996. 
(46) Porod, G. Kolloid Z. 1951, 124, 83.

(47) Porod, G. Kolloid Z. 1952, 125, 51.

(48) Fernández-Barbero, A.; Fernández-Nieves, A.; Grillo, I.; Lopez-Cabarcos, E. Phys Rev E 2002, 66, 051803.

(49) Bastide, J.; Candau, S. J. in Physical Properties of Polymeric gels; Cohen Addad, J. P., ed.; Wiley: Chichester, U.K., 1996.

(50) Brochard, F.; de Gennes, P. G. Macromolecules 1977, 10, 1157.

(51) Hecht, A. M.; Duplessix, R.; Geissler, E. Macromolecules 1985, $18,2167$.

(52) Daoud, M.; Cotton, J. P.; Farnoux, B.; Jannink, G.; Sharma, G.; Benoit, H.; Duplessix, R.; Picot, C.; de Gennes, P. G. Macromolecules 1975, 8, 804.

(53) Bastide, J. in Physical Properties of Polymeric gels; Cohen Addad, J. P., ed.; Wiley: New York, 1996.

(54) Kratz, K.; Hellweg, T.; Eimer, W. Polymer 2001, 42, 6631.

(55) Pedersen, J. S.; Posselt, D.; Mortensen, K. J. Appl. Crystallogr. 1990, 23, 321.

(56) Tzvetkov, G.; Graf, B.; Fernandes, P.; Fery, A.; Cavalieri, F.; Paradossi, G.; Fink, R. Soft Matter 2008, 4, 510.

(57) Fernandes, P.; Tzvetkov, G.; Fink, R.; Paradossi, G.; Fery, A. Langmuir 2008, 24, 13677.

(58) Tzvetkov, G.; Fernandes, P.; Wenzel, S.; Fery, A.; Paradossi, G.; Fink, R. Phys. Chem. Chem. Phys. 2009, 11, 1098.

(59) Tzvetkov, G.; Paradossi, G.; Tortora, M.; Fernandes, P.; Fery, A.; Graf-Zeiler, B.; Fink, R. Mater. Sci. Eng., C 2010, 30, 412.

(60) Fujji, S.; Armes, S.; Araki, T.; Ade, H. J. Am. Chem. Soc. 2005, 127, 16808.

(61) Fujji, S.; Dupin, D.; Araki, T.; Armes, S.; Ade, H. Langmuir 2009, 25, 2588.

(62) Crowther, H. M.; Saunders, B. R.; Mears, S. J.; Cosgrove, T.; Vincent, B.; King, S. M.; Yu, G.-E. Colloids Surf., A 1999, 152, 327. 